Ann. Génét. Sél. anim., I973, 5 (2), 23I-238.

\title{
REMARQUES SUR LA RENTABILITÉ DE LA SÉLECTION ( $\left.{ }^{1}\right)$
}

\author{
M. POUTOUS \\ Station de Génétique quantitative et appliquée, \\ Centre national de Recherches zootechniques, I. N. R. A., \\ 78350 Jouy en Josas
}

\section{RÉSUMÉ}

Toute opération de sélection entraîne des coûts et procure des recettes supplémentaires. Cet article décrit sommairement les principaux postes de coût et de gain, pour un ensemble d'éleveurs rassemblés dans un centre de sélection.

Les frais et les gains n'ayant pas lieu à la même date, on ne peut les introduire dans une même expression que s'ils sont rendus comparables. Les méthodes d'actualisation qui sont décrites ici permettent d'arriver à ce résultat. Il est ainsi possible de rechercher les conditions rendant la sélection la plus rentable possible.

Les conditions sub-optimales sont souvent préférables. Pour une diminution de rentabilité faible, elles permettent de tenir compte de considérations autres que celles du calcul, par exemple des limites techniques ou financières propres à chaque cas.

\section{I. - INTRODUC'TION}

Jusqu'à une date récente, la plupart des travaux en sélection animale ont porté sur l'étude des causes de variation des productions et des méthodes d'expression de la valeur des reproducteurs. La diffusion de l'insémination artificielle puis la possibilité de conserver le sperme sur une longue durée fait que ce genre de recherche, s'il reste toujours indispensable, ne suffit plus. Car, tout au moins pour les reproducteurs mâles de l'espèce bovine, la sélection tend de plus en plus à se faire par un nombre réduit d'entreprises. Par exemple pour la France, nous ne pensons pas qu'il restera plus d'une dizaine de centres de production de semence d'ici quelques années. Une telle concentration des centres de décision et des moyens économiques est, en

(1) Rapport présenté à la Réunion annuelle de la Fédération européenne de Zootechnie, Session commune de la Commission de Génétique et de la Commission de Production bovine, Vérone, Italie le 7 octobre I972. 
principe, favorable à la sélection, mais une erreur technique importante n'est plus tolérable tant pour la firme elle-même que pour les nombreux éleveurs qui lui font confiance. Ceci implique que l'ensemble des opérations du centre de sélection fasse l'objet d'un plan rationnel et cohérent. Les travaux de ROBERTSON (I957) me paraissent, de ce point de vue, importants. Ils ont montré que les effets de la sélection pouvaient être représentés par un petit nombre de formules mathématiques, assez simples pour une utilisation pratique. Ils ont également mis en évidence que les relations entre les principaux paramètres sont parfois complexes et difficiles à établir intuitivement. Enfin, les valeurs optimales existent et varient, dans des limites plus ou moins grandes avec les hypothèses acceptées par l'expérimentateur etles contraintes pratiques qui lui sont imposées.

Il était donc possible d'établir un plan de sélection. Mais une solution purement génétique n'était pas acceptable dans les conditions françaises des années 55-58. Les centres d'insémination qui avaient compris l'intérêt de la sélection sur descendance étaient peu nombreux et devaient financer la plus grande partie des opérations, en particulier les contrôles des performances très peu développés à cette époque. Ils devaient donc demander à leurs adhérents des cotisations plus importantes que les Centres concurrents. Par conséquent, la préoccupation essentielle devait être d'estimer les conditions optimales de la rentabilité économique des sommes investies dans la sélection, compte tenu des contraintes psychologiques, techniques et financières du moment. C'est ce que nous avons essayé de faire avec VISSAC (I962).

Ce thème a déjà fait l'objet de plusieurs publications de nature analytique (cf. bibliographie). Durant cette session, plusieurs rapports seront également présentés. Aussi, notre exposé sera simplifié.

Il fait apparaître que tout programme de sélection implique des coûts à court terme et des recettes à plus long terme. Le problème est de se placer dans les conditions où les coûts sont rentabilisés au mieux. Du point de vue économique, nous avons seulement insisté sur les techniques d'actualisation qui permettent des comparaisons pour des opérations échelonnées dans le temps. Il est évident, par ailleurs, qu'une étude économique complète se ferait à 3 niveaux au moins : l'éleveur pris isolément, le centre de sélection, la collectivité nationale. Leurs intérêts à court terme ne sont pas forcément concordants, de même que leurs possibilités de raisonner à long terme. Nous avons admis ici une similitude suffisante d'objectifs entre le centre de sélection et ses adhérents pour pouvoir les traiter comme une unité.

\section{II. - LES TECHNIQUES D'ACTUALISATION}

Prenons le cas d'une opération de sélection qui débute. Le problème posé est, en principe, assez simple puisqu'il s'agit d'établir, pour une situation donnée, les coûts pour le Centre de sélection et les revenus de ses adhérents. A remarquer que nous admettons implicitement une concordance étroite entre les intérêts de la firme de sélection et ceux de ses utilisateurs, concordance qui n'est pas toujours évidente. Car la structure psychologique et économique des frais et des gains n'est pas du tout la même. Les premiers sont engagés sur une période de temps relativement courte et constituent une sortie réelle et visible de capitaux. Les gains, au contraire, ne 
sont qu'une espérance et ne se réaliseront que plusieurs années après (de l'ordre de IO-I3 ans pour la production laitière). En d'autres termes, les dépenses sont réelles et concentrées dans le temps ; les gains sont prévisionnels et reportés à plusieurs années. Bien entendu, la séparation est moins nette lorsque la sélection est plus avancée et que, par exemple, l'éleveur paye plus cher pour avoir de la semence de bons taureaux mais qu'il voit la production de ses génisses filles des bons taureaux d'il y a 3 ou 4 ans De toute façon, les gains sont d'autant mieux conçus comme tels que leur échéance de réalisation est proche. Une pondération, en raison inverse du temps écoulé jusqu'à la réalisation de l'événement, apparaît alors indispensable.

Bien entendu, ces motifs psychologiques sont étayés par des considérations de nature économique. Car aussi bien la firme de sélection que la collectivité nationale qui assume une partie des dépenses doivent tenir compte de l'échelonnement dans le temps des dépenses et des recettes pour établir les conditions optimales de rentabilité d'un projet ou bien pour choisir entre différents projets. Il faut un moyen pour rendre comparables des mouvements de capitaux réalisés chaque année depuis le début jusqu'à la fin du projet. Ia technique employée généralement est 1'actualisation, dont une bonne description est donnée par SoLLER et al. (I966) et HILL (I97I). Brièvement on peut la résumer ainsi :

Soit $a_{0}$ la somme empruntée l'année initiale et $t$ le taux d'intérêt annuel, par le jeu des intérêts composés la somme due l'année $n$ sera :

$$
a_{n}=a_{0}(\mathrm{I}+t)^{n}
$$

Inversement une somme $A_{n}$ empruntée l'année $n$ serait équivalente à une somme $A_{0}$ empruntée au début telle que : $A_{n}=A_{0}(\mathrm{I}+t)^{n}$ (d'après (I))

$$
A_{o}=A_{n}\left(\frac{\mathrm{I}}{\mathrm{I}+t}\right)^{n}
$$

Un raisonnement analogue pourrait être tenu pour les rentrées d'argent. Le taux $t$ est appelé taux d'actualisation, le rapport $R_{n}=\left(\frac{\mathrm{I}}{\mathrm{I}+t}\right)^{n}$ étant le facteur d'actualisation. Finalement, toute rentrée ou sortie d'argent enregistrée l'année $n$ peut être convertie en somme équivalente pour l'année de départ par multiplication par le facteur d'actualisation $R_{n}$. Le choix de l'année de référence est d'ailleurs arbitraire, les résultats étant les mêmes à un coefficient près. Par rapport aux techniques classiques, l'actualisation réduit considérablement l'importance des événements éloignés et ce d'autant plus que le taux d'actualisation est plus élevé. Pour les valeurs habituelles, il suffit d'un intervalle de 4 à 6 ans, à peine une génération, pour réduire de moitié l'importance accordée à des rentrées d'argent. On favorise donc les méthodes qui donnent les résultats les plus rapides.

Un programme de sélection pouvant se dérouler indéfiniment, en général on se fixe une limite au-delà de laquelle on considère que le schéma est terminé. Cette durée d'actualisation agit en sens contraire du taux d'actualisation d'une manière complexe. Il est souvent difficile de prendre une durée trop courte et au-delà de I520 ans les résultats ne sont pas très différents, MOCQUOT et FOULLEY (I973).

Le choix du taux et de la durée d'actualisation sont, dans une large mesure, arbitraires. En particulier, le taux, qui ne devrait normalement pas descendre en dessous du taux d'intérêt commercial, est souvent abaissé pour des motifs de poli- 
tique générale d'un pays ou bien parce que la collectivité estime nécessaire de développer une production donnée même à un prix relativement élevé. Inversement, on peut choisir un taux élevé pour essayer de tenir compte de fluctuations imprévisibles tant sur les coûts et les bénéfices que sur les besoins futurs du marché et les techniques de sélection. Quant à la durée d'actualisation, elle n'a pratiquement plus d'influence au-delà d'une vingtaine d'années, mais la collectivité peut parfois s'intéresser à l'état du cheptel après ce délai.

Malgré ses imperfections, l'actualisation est, sans doute, une des techniques économiques les plus simples pour tenir compte de l'échelonnement dans le temps des opérations de sélection. Ceci est particulièrement important dans le cas de la production laitière où l'intervalle de génération est très élevé de sorte que le court terme (8-Io ans) serait déjà un long terme pour la plupart des activités industrielles.

\section{III. - EXPRESSION DES RECE'TTES}

I. Les recettes sont à estimer pour chaque année $(n)$ et à actualiser sur l'année de référence. Pour un caractère, on peut écrire :

$$
B_{n}=N_{n} \quad G_{n} \quad V_{n} R_{n}
$$

avec $B_{n}=$ recettes actualisées pour l'année $n, N_{n}$ nombre de performances, $G_{n}$ supériorité génétique moyenne des performances par rapport à l'année de départ, $V_{n}$ bénéfice unitaire, $R_{n}$ facteur d'actualisation. C'est évidemment dans l'évaluation de ces paramètres qu'on doit faire intervenir un grand nombre d'hypothèses.

$N_{n}$ : Le nombre de performances est très simple à calculer dans le cas d'animaux croisés utilisés pour la boucherie. Partout ailleurs, il découle du nombre d'inséminations, des valeurs admises pour l'élimination aux différents stades, taux de fécondation, pourcentages d'animaux arrivant à la $\mathrm{I}^{\mathrm{re}}, 2^{\mathrm{e}}$, etc., mise bas, et de la probabilité d'obtenir des descendants à la $2^{e}$, etc., génération. En production laitière, i1 s'écoule pratiquement ro ans avant qu'un mâle retenu influence le niveau génétique de la population femelle, sa contribution pour les années suivantes étant faible mais constante (HINks, I97I).

$G_{n}$ : La supériorité génétique moyenne peut se calculer d'après des techniques devenues classiques (LINDHE, I968). Ici encore, on doit admettre de nombreuses hypothèses, en particulier sur la réponse vraie à la sélection pour des intensités très élevées de sélection qui conduisent à des effectifs génétiques vrais peu élevés. Dans le cas de sélection indirecte, les résultats dépendent assez étroitement des corrélations génétiques, corrélations généralement estimées avec une très faible précision ou variables dans le temps et l'espace (MOCQUOT et FOULLEY, I973).

$V_{n}$ : Estimer le bénéfice unitaire pose évidemment beaucoup de problèmes. Par souci de simplification, on admet généralement qu'il reste constant dans le temps, ou tout au moins qu'il varie proportionnellement aux frais. On admet également qu'il reste le même quel que soit le niveau de production ce qui n'est sans doute pas toujours vrai (par ex. passage d'une alimentation à l'herbe à une alimentation en concentrés pour de fortes productions laitières).

2. Logiquement, il faudrait faire intervenir dans 1'expression des recettes et 
des coûts tous les caractères ayant un intérêt économique. Cependant, on tient compte en général, d'un seul caractère, ou de deux dans le cas de sélection combinée laitvitesse de croissance. Ceci revient à admettre des corrélations génétiques nulles entre le caractère à améliorer et les autres. L’interprétation des résultats est donc à faire avec prudence soit pour estimer les effets à long terme de la sélection soit pour juger de l'intérêt d'une substitution de race.

Il me semble également que, tout au moins dans certaines conditions, il existe des contraintes sévères dans le nombre d'animaux contrôlés et, parmi ces derniers, dans l'effectif disponible pour la mise à l'épreuve des jeunes reproducteurs. Il serait trop long d'insister sur ce problème qui est, cependant, bien réel en France. Nous voudrions seulement faire remarquer qu'on devrait en tenir compte dans le choix de la méthode de sélection mais aussi de contrôle des performances et que les arguments techniques ne seraient alors pas les seuls à prendre en considération.

\section{IV. - EXPRESSION DES COÛTS}

Les frais supplémentaires sont de 3 ordres :

a) Frais supplémentaires liés aux animaux producteurs ; nous avons admis qu'ils étaient incorporés dans l'expression des bénéfices unitaires.

b) Frais de mise à l'épreuve sur descendance des reproducteurs mâles. La prime versée aux éleveurs est fonction du nombre de femelles contrôlées, de l'opinion des éleveurs sur la valeur des jeunes mâles à tester et de la disponibilité et du prix de la semønce des taureaux testés. En France tout au moins, la prime de testage n'est pas négligeable puisqu'elle équivaut à environ 300 à 500 litres de lait. Les dépenses globales pour les contrôles de performances sont encore plus élevés. Incidemment, cєci justifie des études méthodiques sur l'efficacité réelle pour la sélection et la gestion des troupeaux, des contrôles de performances dans leur forme actuelle etleurs variantes possibles.

c) Les frais liés aux mâles comprennent l'achat, les contrôles, l'entretien aux divers stades de la vie du reproducteur, enfin la collecte, le stockage et l'utilisation de la semence. Pour simplifier les calculs, ces frais ont été évalués forfaitairement par la plupart des auteurs, ce qui rend les comparaisons difficiles. Il nous paraît pourtant évident, qu'il y aurait souvent des économies à faire, par exemple sur le type ce bâtiments ou la conduite des animaux. Quoi qu'il en soit, dans un schéma réel, il faut au moins mettre à part les investissements immobiliers (bâtiments, terres, etc.) parce qu'ils sont souvent lourds, qu'ils doivent être réalisés en début d'opération, qu'ils varient de façon discrète (pour les bâtiments tout au moins) et que leur valeur future d'inventaire est évidemment difficile à évaluer.

\section{V. - EXPRESSION DE LA RENTABILITÉ}

Si le ou les programmes de sélection examinés sont totalement définis, la rentabilité s'exprime simplement par la différence des recettes et des coûts, après actualisation. En général, il s'agit plutôt de rechercher les valeurs optimales pour les 
paramètres qu'on ne se fixe pas a priori, par exemple, les intensités de sélecticn. La façon dont ces valeurs sont obtenues ne présente pas d'importance en soi, qu'il s'agisse de dérivation algébrique approximative (HILL, I97I) ou de calculs par points (LINDHE, I966; MocQuOT et FoulLEY, I973). La présentation des résultats n'est pas facile lorsque au moins deux paramètres sont libres. Pour faciliter l'interprétation par les utilisateurs, il apparait intéressant de choisir une seule variable indépendante tenant compte de toutes les autres. Le volume des dépenses est souvent choisi du fait de son importance économique, d'un point de vue plus génétique le progrès espéré pourrait jouer un rôle analogue (MocQuOT et FoULLEY, I973).

En pratique, les valeurs strictement optimales ne sont pas toujours celles à rechercher car, elles peuvent conduire à des impossibilités pratiques (ex : nombre de mâles), ou dépasser la zone de validité des hypothèses de travail, ou risquer d'entraîner à long terme des conséquences défavorables sur d'autres caractères que celui considéré dans la sélection. C'est pour ces raisons qu'on recherche souvent une zone de variation suboptimale, qui est semble-t-il assez large (PouTous, VisSAC, I962), les valeurs adoptées pour les paramètres dans cette zone pouvant faire intervenir d'autres considérations que la rentabilité. D'un autre point de vue, une telle zone permet d'estimer l'incidence sur les résultats d'une variation autour de l'optimum et cette variation est inévitable en pratique.

\section{VI. - DISCUSSION ET CONCLUSION}

Ces calculs reposent sur des méthodes économiques simples. Au moins dans un premier stade, ceci ne nous paraît pas un inconvénient bien grave. Il s'agissait d'abord de montrer que la sélection pouvait se raisonner en termes économétriques. Ce point étant maintenant bien admis, peut-être une analyse plus fine serait-elle utile, à condition cependant de ne pas aboutir à un modèle tellement complexe qu'il deviendrait inutilisable.

Le choix des conditions d'actualisation est important. Cependant ce n'est pas le sélectionneur qui les fixe; tout au plus peut-on montrer qu'à des valeurs différentes correspondent des méthodes de sélection et une rentabilité différentes (Mocquot et FOULLEY, I973). Cependant, il paraît raisonnable d'accorder une certaine préférence à la méthode la moins sensible à des variations, au moins modérées, des conditions d'actualisation. Ainsi les fluctuations inévitables, compte tenu des délais de la sélection, n'aurait pas d'incidence catastrophique. Cette méthode de calcul pour des valeurs discrètes des paramètres a d'ailleurs été appliquée par la plupart des aut pour les paramètres génétiques qui varient dans le temps comme les héritabilités ou dont les valeurs sont mal connues, comme c'est souvent le cas pour les corrélations génétiques. Il est également possible de faire varier les coûts et les bénéfices unitaires. Il ne faut cependant pas se dissimuler la difficulté de prévoir l'évolution des besoins de façon précise, car, comme l'a montré Hinks, le délai d'attente des effets de la sélection est très long. Peut-être, ici aussi, faudrait-il favoriser les méthodes efficaces dans les conditions prévisibles mais permettant, avec un délai assez court, une adaptation à des objectifs de sélection non prévus à l'heure actuelle. 
L'application directe des résultats obtenus par les auteurs cités en bibliographie doit, nous semble-t-il, être faite avec beaucoup de prudence. Car les conditions locales peuvent être très différentes et, par ailleurs, la méthodologie employée n'est pas toujours la meilleure possible. Cependant, ces études montrent bien l'intérêt de la méthode qui a permis, par exemple, à Mc Clintock et Cunningham de montrer qu'un fort pourcentage de croisement industriel améliore la sélection laitière. Ce résultat d'apparence paradoxale peut être discuté ; il a au moins le mérite de reposer un vieux problème en des termes nouveaux et rationnels. Il reste beaucoup à faire, mais dès à présent, il apparaît possible de raisonner la sélection en termes économiques sur des bases objectives. Sous réserve de ne pas oublier les imperfections du modèle et de maintenir une grande souplesse d'application, il s'agit là d'un progrès très appréciable.

Reçu pour publication en novembre 1972.

\section{SUMMARY}

\section{NOTE ON SELECTION RENTABILITY}

All selection operations involve cost and result in additional gain. This article briefly describes areas of cost and gain for all the breeders grouped in a selection center.

Since cost and gain do not occur at the same date, they can only be introduced in the same expression if they are made comparable. This result may be achieved using methods of realization described here, thus making it possible to study conditions which make selection maximally profitable.

Sub-optimal conditions are often preferable. For low gain, they take into account considerations other than those of computation, as for example technical or financial limits of each individual case.

\section{ZUSAMMENFASSUNG}

\section{BEMERKUNGEN ZUR ZUCHT-RENTABILITÄT}

Jedes Zuchtgeschehen ist einerseits mit Kosten verbunden und führt andererseits zu zusätz lichen Erträgen. In dieser Arbeit werden summarisch die einzelnen Kosten- und Gewinnstellen beschrieben, bezogen auf die Gesamtheit der Züchter eines Zuchtzentrums.

Da die Kosten und Gewinne nicht im gleichen Zeitpunkt auftreten, können sie nur in der selben Gleichung eingeführt werden, wenn sie vergleichbar gemacht werden. Die in der Arbeit beschriebenen Methode, die Kosten und Gewinne auf den aktuellen Zeitpunkt zu beziehen, führt zur gewünschten Vergleichbarkeit. Sie ermöglicht, die für die Zucht rentabelsten Bedingungen $\mathrm{zu}$ bestimmen.

Oftmals sind zur Erhöhung der Rentabilität suboptimale Bedingungen vorzuziehen. Sie gesttaten andere als rechnerisch erfassbare Gegebenheiten, wie, zum Beispiel, technische oder finanzielle Begrenzungen, die für einen bestimmten Fall gelten, zu berücksichtigen.

\section{RÉFÉRENCES BIBLIOGRAPHIQUES}

Hrll W. G., I971. Investment appraisal for national programmes. Anim. Prod., 13, 37-50.

Hinks C. J. M., I970 a. The selection of dairy bulls for AI Anim. Prod., 12, 569-576.

Hinks C. J. M., r97o $b$. Performance test procedures for meat production amongt dairy bulls used in A. I. Anim. Prod., 12, 577-583. 
Hinks C. J. M., I97r. The genetic and financial consequences of selection amongst dairy bulls in artifical insemination. Anim. Prod., 13, 209-2 I8.

Lindhe B., r968. Model simulation of A. I. Breeding within a dual purpose breed of cattle. Acta Agr. Scand., 18, 33-41.

Mc Clintock A. E., Cunningham E. P., I97i. AI breeding strategy for dual purpose cattle populations. Ann. Génét. Sél. anim., 4, I37-138.

Mocouot J.-C., Foulley J.-L., r973. Recherche des conditions de rentabilité d'un schéma de sélection d'une souche de bovins destinés au croisement de première génération pour la production de veaux de boucherie. Ann. Génét. Sél. anim., 5 I89-209.

Poutous M., Vissac B., 1962. Recherche théorique des conditions de rentabilité maximum de l'épreuve de descendance des taureaux d'insémination artificielle. Ann. Zootech., 11, 233-256.

Soller M., Bar-ANan R., Pasternak H., I966. Selection of dairy cattle for growth rate and milk production. Anim. Prod., 8, rog-I Ig. 